\title{
Effects of Lactoalbumin Addition to Vulcanized Rubber Determined by RPA2000
}

\author{
Jiao Yang ${ }^{1,2, a}$, Tianming Gao ${ }^{2, b}$, Hongxing Gui ${ }^{2, c}$, Jianfeng Guo ${ }^{1, d}$, Maofang \\ Huang ${ }^{2, f}$ \\ ${ }^{1}$ Chemical Industry and Ecology Institute,North University of China, Taiyuan 030051,China \\ ${ }^{2}$ Agricultural Products Processing Research Institute,Chinese Academy of Tropical Agricultural \\ Science,Zhanjiang 524001,China; \\ a253558538@qq.com, ${ }^{b} 38020137 @ q q . c o m,{ }^{c}$ guihongxing@236.net, \\ dshfguojianfeng@qq.com,'huangmaofang666@163.com
}

Keywords: vulcanized rubber lactoalbumin RPA

\begin{abstract}
We investigated the effect of lactoalbumin on the processing properties of vulcanized rubber by adding lactoalbumin protein. We found that G' and G' of vulcanized rubber were increased and $\tan \delta$ was decreased by adding lactoalbumin. The addition of lactoalbumin can improve the processing properties of natural rubber.
\end{abstract}

\section{Introduction}

RPA2000 is a fast multi-purpose rubber processing analyzer, but also a new kind of dynamic mechanical rheometer.It can characterize the properties of raw rubber, vulcanized rubber processing properties, vulcanized rubber physical properties, and curing properties. It is a powerful tool for research and production quality control of rubber products.[1,2]

In this paper, we investigated the effect of lactoalbumin addition on the properties of vulcanized rubber by using RPA2000 to monitor the elastic modulus, viscous modulus and loss factor.

\section{Experiment}

\section{Materials}

The natural rubber(NR)was supplied by Gaozhou Rubber Processing Center (MaoMing,China).

Lactoalbumin, sulfur, accelerator NS, zinc oxide and stearic acid were commercially available industrial products.

\section{Methods}

Preparation of the samples

Different amounts of lactoalbumin were added to a certain amount of NR. Lactoalbumin were added, according to Table 1 , to obtain the samples.We used 3\% acetic acid to allow solidification, and then tableted, pelletized, soaked, and dried the samples to obtain natural rubber (NR). 
Table 1 Formulation for the Samples

\begin{tabular}{cc}
\hline Sample & Lactoalbumin(\%) \\
\hline 1 & 0 \\
2 & 2 \\
3 & 4 \\
4 & 6 \\
5 & 8 \\
\hline
\end{tabular}

\section{Preparation of the vulcanized samples}

In a mixing machine,ingredients were added to NR .according to the following formula (w/w): sample,100; stearic acid, 0.5; sulfur, 3.5; NS, 0.5; and ZnO,6.0,to obtain gross rubber.

\section{Measurement and Characterization}

Rubber Process Analyzer(RPA)

The RPA of vulcanized rubber was carried out with a RPA2000 rubber process analyzer (ALPHA, United States).

The temperature scan was performed at a frequency of 6CPM, strain of 1Deg, and the testing temperature ranged from $60^{\circ} \mathrm{C}$ to $160^{\circ} \mathrm{C}$.

The strain scan was performed at a frequency of $6 \mathrm{CPM}$, temperature of $60^{\circ} \mathrm{C}$, and the testing stain ranged from 0.02Deg to 30Deg.

The frequency scan was performed at a strain of $1 \mathrm{Deg}$, temperature of $60^{\circ} \mathrm{C}$, and the testing frequency ranged from 2CPM to 2000CPM.

\section{Results and discussion}

\section{Strain scanning}

Strain sweeping was carried out on the vulcanized rubber at $6 \mathrm{CPM}$ and $60^{\circ} \mathrm{C}$,. The relationships between the elastic modulus $G^{\prime}$, the viscous modulus $G$ ", loss factor ( $\tan \delta$ ) and strain are shown in figure 1 3.It can be seen from figure 1 that $G$ ' of vulcanized rubber changes significantly under low strain.As strain increases, $G$ ' drops rapidly.This shows that the molecular structure of vulcanized rubber has undergone significant changes in response to increasing strain,which eventually disappear with linear viscoelasticity.[3]When the strain is more than 200\%, the trend slows significantly. In figure 1, as the amount of lactoalbumin increased, the G' initial value of vulcanized rubber increased,as zinc oxide and accelerator enhance the grid structure of vulcanized rubber.

Under the same strain, increased amounts of lactoalbumin resulted in increased G' of the vulcanized rubber, as shown in figure 2. The addition of lactoalbumin and Curing Formulations causes significant changes. Researching the dynamic performance and differences oinvulcanized rubber over different strain conditions is useful to understand the structure and characteristics and processing performance under actual manufacturing condition .[4]

As can be seen from figure 3 , tan $\delta$ increases obviously as strain increases. The tan $\delta$ is the ratio of the G 'and' G ', and indicates that the G' response to strain is greater than the $G$ ' response to strain. At the same degree of strain, the more the amount of lactoalbumin, the greater the $\tan \delta$. 


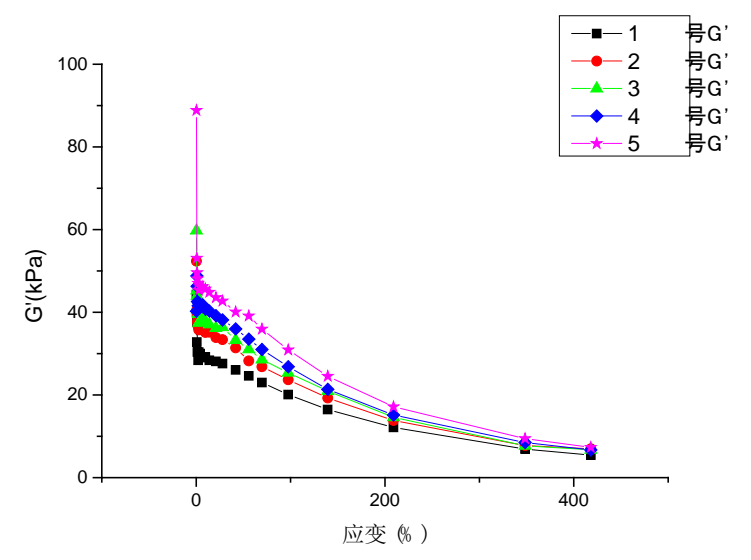

Fig.1 Vulcanized rubber sample G' 's response to the variation of stresses

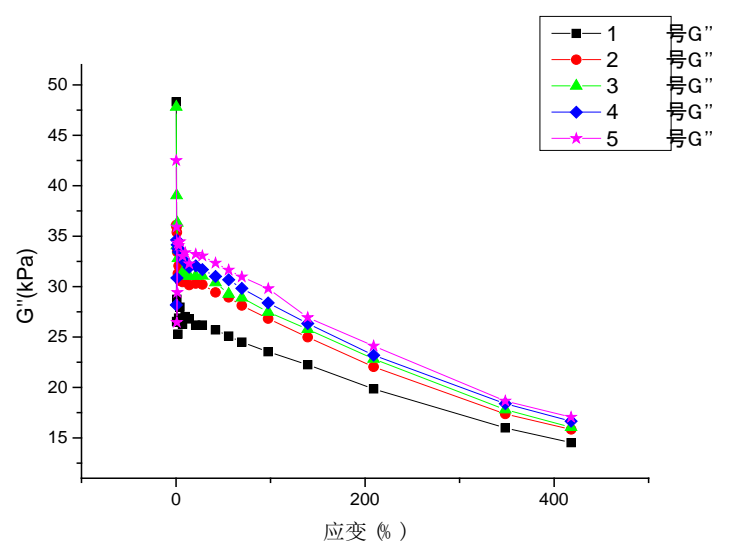

Fig.2Vulcanized rubber sample G”' 's response to the variation of stresses

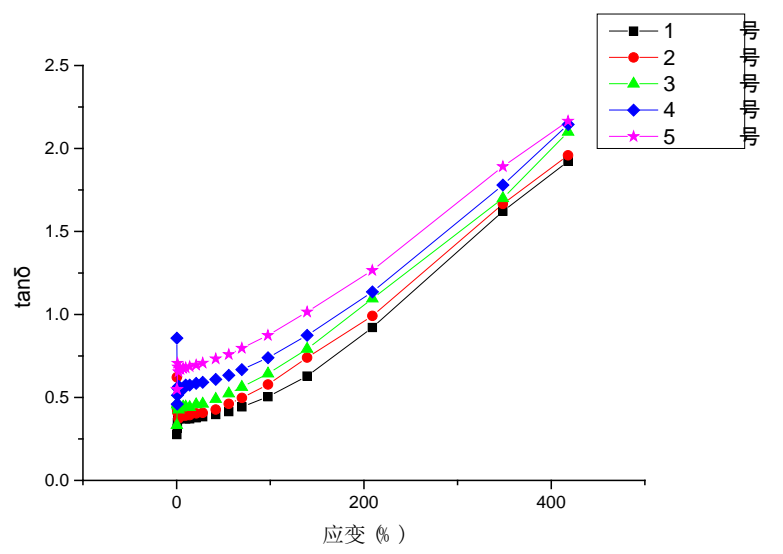

Fig.3 Vulcanized rubber sample tan $\delta$ 's response to the variation of stresses

\section{Frequency scanning}

At $60^{\circ} \mathrm{C}$ and $1 \mathrm{Deg}$ in stain, frequency scanning was carried out on the vulcanized rubber as shown in figures 4-6.As frequency increases, the elastic modulus $G^{\prime}$ increases. When the frequency was increased to $1000 \mathrm{CPM}$, the amplitude decreased and gradually stabilized. As lactoalbumin concentration increased, the elastic modulus $G$ ' increased. This may be due to changes in intermolecular grid structure during the curing process.In frequency scanning of vulcanized rubber, the greater the G' more liquidity, which leads to an increase in $\tan \delta$.

The viscous modulus G" increased rapidly with the increase in frequency. When frequency reached 200 CPM, the viscous modulus G' became stable. At the same frequency, the viscous 
modulus G” and rising amplitude increased with increased lactoalbumin conentration.

The loss factor tan $\delta$ of vulcanized rubber decreased rapidly and then stablilized with the increase of frequency. The downward trend was less dramatic with increased lactoalbumin.

Loss factor $\tan \delta$ indicates the quality of processing properties[5].Frequency scanning can characterize the speed level of processing, and processing properties decrease if speed is too fast.[6] However, the reducing effect of the frequency will be reduced by the addition lactoalbumin. Therefore, addition of the correct amount of lactoalbumin can improve the processing performance of rubber.

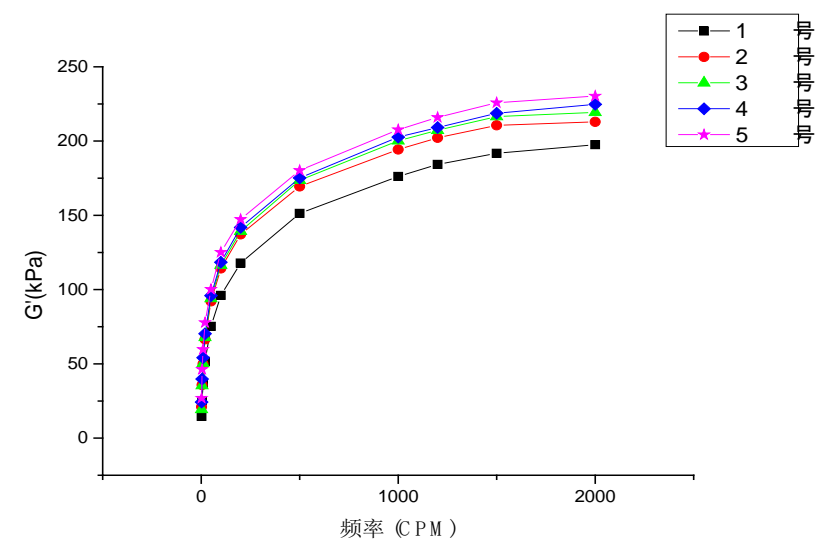

Fig.4 Vulcanized rubber sample G' 's response to frequency

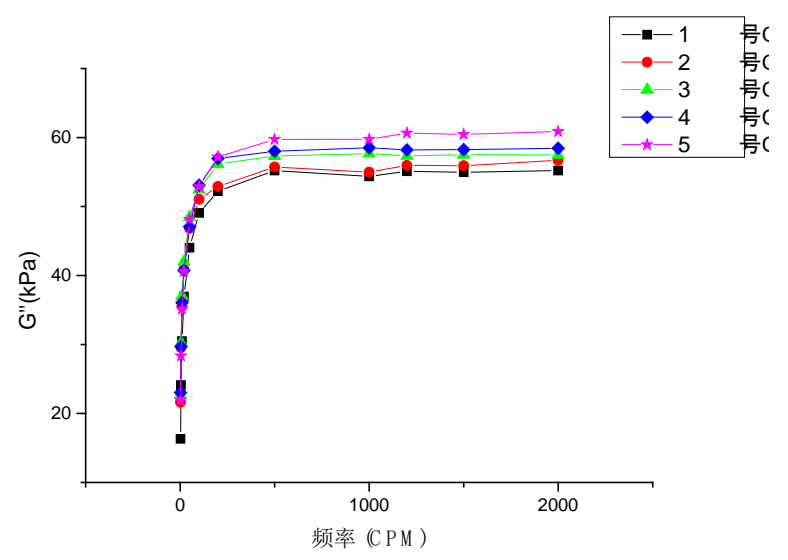

Fig.5 Vulcanized rubber sample G”' 's response to frequency

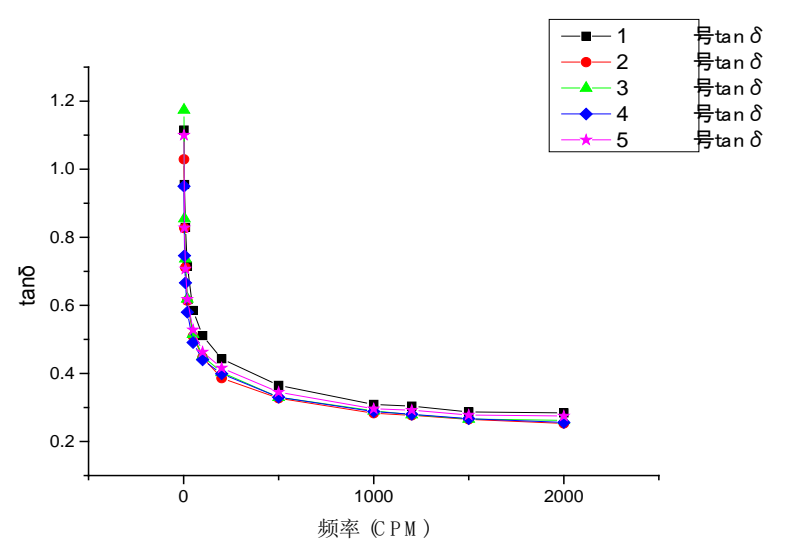

Fig.6 Vulcanized rubber sample tan $\delta$ 's response to frequency

\section{Temperature scanning}

Using 6CPM in frequency and 1Deg in strain, temperature scanning of vulcanized rubber was 
eofromed and the results are shown in figures 7 and 9. The elastic modulus G' first decreased with the increase of temperature, and then once the temperature reached $150{ }^{\circ} \mathrm{C}$, it rapidly increased. This is likely because raising the temperature resulted in increased motility of vulcanized rubber molecules and the weakening of resistance to deformation force and decreased G'.[7]The initial values of G' was increased with increased lactoalbumin concentration, and the curve descent velocity was essentially identical. The viscous modulus G” of vulcanized rubber decreased as temperature increased and had a linear relation with the amount of lactoalbumin. The loss factor $\tan \delta$ increased at first and then decreased with further temperature increase. When the temperature reached $120{ }^{\circ} \mathrm{C}$, the tan $\delta$ was maximum. The starting value was reduced and the trend changes occured essentially at the same steps as lactoalbumin increased.

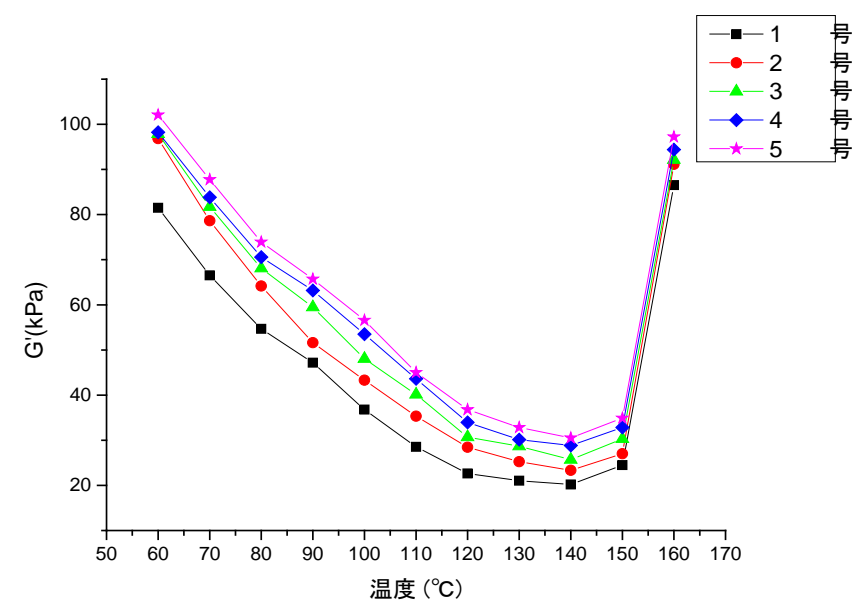

Fig.7Vulcanized rubber sample G' 's response to temperature

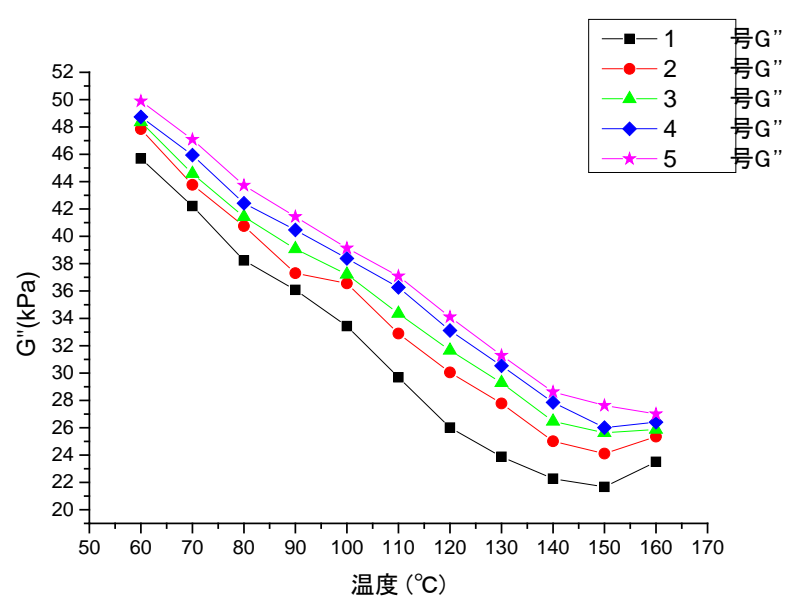

Fig.8 Vulcanized rubber sample G”'s response to temperature 


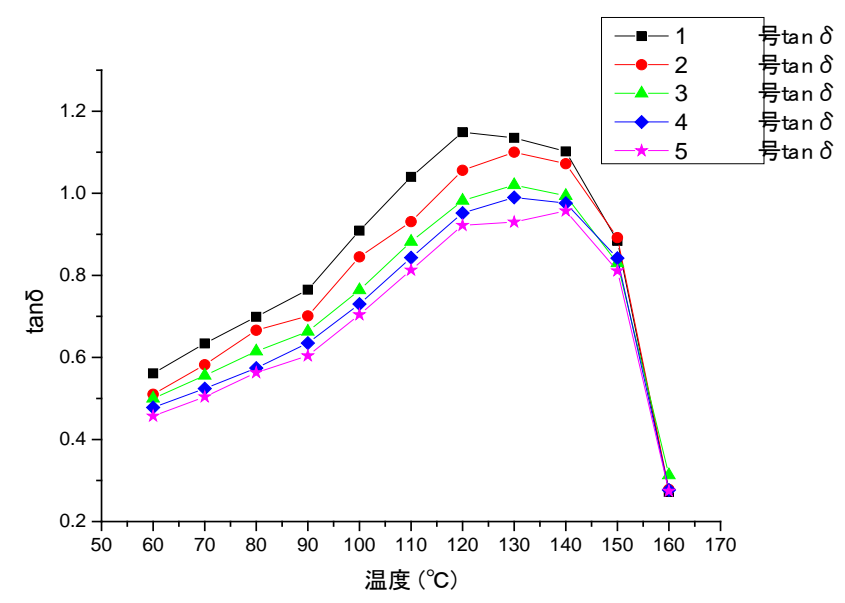

Fig.9 Vulcanized rubber sample tan $\delta$ 's response to temperature

\section{Conclusions}

(1)The G' and G" of vulcanized rubber was gradually reduced, and tan $\delta$ was gradually increased with increased strain; lactoalbumin helped to improve the G' and G'”

(2)The G' and G' of vulcanized rubber was gradually increased, and tan $\delta$ was gradually reduced with the increase of frequency. At the same frequency,lactoalbumin improved the $G^{\prime} 、 G$ ' and $\tan \delta$ of vulcanized rubber.

(3)With increased temperature, the G' and G', of vulcanized rubber decreased. However, the G' and $\tan \delta$ of vulcanized rubber made a rebound when the temperature exceeded $150{ }^{\circ} \mathrm{C}$.

In conclusion, adding lactoalbumin improved the processing properties of vulcanized rubber and holds potential to extend the application field of natural rubber.

\section{References}

[1]Zhongtie Su,etc.Detection of RPA2000 rubber processing analyzer.China Rubber Industry,2002,49 (4): 235-237.

[2] Zeng J,Li WD.Application of rubber processing analyzer RPA2000 to properties of SBR1723 and SBR1712E[J]. Rubber Science and Technology,2010,17:4-10.

[3] Guiyi Wang. Application of rubber processing analyzer RPA2000 to rubber study[J].Special Purpose Rubber Products,2001,22(1): 56-62.

[4] Jo hn S D,etc.Application of rubber processing analyzer RPA2000[J].China Rubber Industry,1998,45(5):301-314.

[5] John AS, Henry AP.New test results from rotor less curemeters[C] .The Rubber Division , American Chemical Society 136th Meeting, 1989-10-17.

[6] Liu D,Wang QF,Song ZB,etc. Study on RPA dynamic properties of different polybutadiene rubber[J].World Rubber Industry, 2009,36 (6): :19-24.

[7] Zeng CR. Polymer molecular weight and its distribution[M].Chemical Industry

Publisher,1986,24-29. 